\title{
Unique solvability for the non-Newtonian magneto-micropolar fluid
}

\section{Changjia Wang*}

\section{"Correspondence:}

wangchangjia@gmail.com

School of Science, Changchun

University of Science and

Technology, Changchun, 130022,

P.R. China

\begin{abstract}
In this paper, a motion of an incompressible non-Newtonian magneto-micropolar fluid is considered. We assume that the stress tensor has a $p$-structure, and we establish the global in time existence and uniqueness of the weak solutions with $p \geq \frac{5}{2}$ in three dimensions.
\end{abstract}

\section{Introduction and main results}

This paper is concerned about the existence and uniqueness of the weak solutions to the non-Newtonian magneto-micropolar fluid equations in $\Omega \times(0, T)$, which are described by

$$
\left\{\begin{array}{l}
u_{t}+(u \cdot \nabla) u-\operatorname{div}\left(|\boldsymbol{e}(u)|^{p-2} \boldsymbol{e}(u)\right)+\nabla\left(\pi+\frac{1}{2}|b|^{2}\right)=\chi \operatorname{rot} \omega+(b \cdot \nabla) b \\
\omega_{t}+(u \cdot \nabla) \omega-\mu \Delta \omega+2 \chi \omega=\chi \operatorname{rot} u \\
b_{t}-\lambda \Delta b+(u \cdot \nabla) b-(b \cdot \nabla) u=0 \\
\operatorname{div} u=\operatorname{div} b=0
\end{array}\right.
$$

here $\Omega \in R^{3}$ is an open-bounded domain with Lipschitz boundaries, and the unknowns $u$, $\omega, b, \pi$ denote the velocity of the fluid, the micro-rotational velocity, magnetic field and hydrostatic pressure, respectively. $\chi, \mu, \lambda$ are positive numbers associated with properties of the material: $\chi$ is the vortex viscosity, $\mu$ is spin viscosity and $\frac{1}{\lambda}$ is the magnetic Reynold. In (1.1), $\boldsymbol{e}(u)$ is the symmetric part of the velocity gradient, i.e.,

$$
2 \boldsymbol{e}(u)=\nabla u+(\nabla u)^{T}
$$

To (1.1) we append the following initial and boundary conditions

$$
\begin{aligned}
& u(x, 0)=u_{0}(x), \quad \omega(x, 0)=\omega_{0}(x), \quad b(x, 0)=b_{0}(x), \quad \forall x \in \Omega, \\
& u(x, t)=\omega(x, t)=b(x, t)=0, \quad \forall(x, t) \in \Sigma_{T},
\end{aligned}
$$

where $\Sigma_{T}=\partial \Omega \times(0, T)$.

The theory of micropolar fluid was first proposed by Eringen [1] in 1966, which enabled us to consider some physical phenomena that cannot be treated by the classical NavierStokes equations for the viscous incompressible fluid, for example, the motion of animal blood, liquid crystals and dilute aqueous polymer solutions, etc.

() 2013 Wang; licensee Springer. This is an Open Access article distributed under the terms of the Creative Commons Attribution License (http://creativecommons.org/licenses/by/2.0), which permits unrestricted use, distribution, and reproduction in any medium, provided the original work is properly cited. 
If $p=2$, system (1.1) reduces to the classical magneto-micropolar fluid equations, and there are many earlier results concerning the weak and strong solvability in a bounded domain $\Omega \in R^{3}$. For strong solutions, Galdi and Rionero [2] stated, without proof, the results of existence and uniqueness of strong solutions. Rojas-Medar [3] studied it and established the local in time existence and uniqueness of strong solutions by the spectral Galerkin method. In 1999, Ortega-Torres and Rojas-Medar [4] proved global existence of strong solutions with the small initial values. For weak solutions, Rojas-Medar and Boldrini [5] proved the existence of weak solutions, and in the 2D case, also proved the uniqueness of the weak solutions.

On the other hand, there are few existence results about the non-Newtonian magnetomicropolar fluid, i.e., the $p \neq 2$ case. In a recent work, Gunzburger et al. [6] studied the reduced problem (with both $\omega=0$ and $\chi=0$ ), and gave the global unique solvability of the first initial-boundary value problem in a bounded two or three-dimensional domain. Improved results are proved for the periodic boundary condition case.

In this paper, we will prove the global existence and uniqueness of the weak solutions for the full system (1.1)-(1.3) under the condition that $p \geq \frac{5}{2}$. These results are based on the Galerkin method and a series of uniform estimates, which do not depend on the parameters.

Throughout this work, we use a standard notation $L^{p}(\Omega)$ (normed $\|\cdot\|_{p}$ ) for Lebesgue $L^{p}$-spaces, as well as $W^{k, p}(\Omega)$ (normed $\|\cdot\|_{k, p}$ ) for the usual Sobolev spaces. As usual, $C_{0}^{\infty}(\Omega)$ denotes the set of all $C^{\infty}$-functions with the compact support in $\Omega$. Given $T>0$ and a Banach space $X$, we denote by $L^{q}(0, T ; X)$ Bochner spaces, which are equipped with the norm

$$
\|\cdot\|_{L^{q}(0, T ; X)}:=\left(\int_{0}^{T}\|\cdot\|_{X}^{q} d s\right)^{\frac{1}{q}} .
$$

We also introduce the following functional vector spaces:

$$
\begin{aligned}
& \mathcal{V} \equiv\left\{u \in C_{0}^{\infty}(\Omega), \operatorname{div} u=0\right\} \\
& H \equiv \text { the closure of } \mathcal{V} \text { in } L^{2}(\Omega), \\
& V_{p} \equiv \text { the closure of } \mathcal{V} \text { in } W^{1, p}(\Omega) .
\end{aligned}
$$

We next introduce the definition of a weak solution for problems (1.1)-(1.3).

Definition 1.1 We say that $(u, \omega, b)$ is a weak solution to problems (1.1)-(1.3) if

$$
\begin{aligned}
& u \in L^{\infty}\left(0, T ; V_{p}\right), \quad u_{t} \in L^{2}(0, T ; H), \\
& \omega \in L^{\infty}\left(0, T ; W^{1,2}(\Omega)\right) \cap L^{2}\left(0, T ; W^{2,2}(\Omega)\right), \\
& b \in L^{\infty}\left(0, T ; V_{2}\right) \cap L^{2}\left(0, T ; W^{2,2}(\Omega)\right), \\
& \omega_{t} \in L^{2}\left(0, T ; W^{1,2}(\Omega)\right), \quad b_{t} \in L^{2}(0, T ; H)
\end{aligned}
$$

satisfy

$$
\begin{aligned}
& \left\langle u_{t}, \phi\right\rangle+\left\langle|\boldsymbol{e}(u)|^{p-2} \boldsymbol{e}(u), \boldsymbol{e}(\phi)\right\rangle=\chi\langle\operatorname{rot} \omega, \phi\rangle+\langle(b \cdot \nabla) b, \phi\rangle-\langle(u \cdot \nabla) u, \phi\rangle \\
& \quad \text { for all } \phi \in V_{p},
\end{aligned}
$$




$$
\begin{aligned}
& \left\langle\omega_{t}, \psi\right\rangle+\mu\langle\nabla \omega, \nabla \psi\rangle=\chi\langle\operatorname{rot} u, \psi\rangle-\langle(u \cdot \nabla) \omega, \psi\rangle-2 \chi\langle\omega, \psi\rangle \\
& \quad \text { for all } \psi \in W_{0}^{1,2}(\Omega) \\
& \left\langle b_{t}, \eta\right\rangle+\lambda\langle\nabla b, \nabla \eta\rangle=\langle(b \cdot \nabla) u, \eta\rangle-\langle(u \cdot \nabla) b, \eta\rangle \\
& \quad \text { for all } \eta \in V_{2}
\end{aligned}
$$

where the symbol $\langle\cdot, \cdot\rangle$ denotes a generic duality pairing.

The following theorem gives the main results of this paper.

Theorem 1.1 Let $\Omega \in R^{3}$ be an open-bounded domain with a Lipschitz boundary $\partial \Omega$. Assume that $p \geq \frac{5}{2}, u_{0} \in W^{1, p}(\Omega), \omega_{0}, b_{0} \in W^{1,2}(\Omega)$. Then, for $\forall T \in(0,+\infty)$, there exists a unique weak solution to problem (1.1)-(1.3) in the sense of Definition 1.1.

Remark 1.1 If (1.4)-(1.5) hold, it could be easy to introduce the pressure $\pi \in L^{\infty}(0, T$; $\left.L^{p^{\prime}}(\Omega)\right), p^{\prime}=p /(p-1)$. This will be done at the end of Section 3 .

For latter use, let us state some useful inequalities.

Lemma 1.1 (See [7]) (Korn's inequality) Let $1<p<\infty$. Then there exists a constant $C_{p}=$ $C_{p}(\Omega)$ such that

$$
C_{p}\|v\|_{1, p} \leq\|\boldsymbol{e}(v)\|_{p} \quad \text { for all } v \in W_{0}^{1, p}(\Omega)
$$

where $\Omega \in R^{n}$ is open and bounded with a Lipschitz boundary.

Lemma 1.2 (See [8]) (On negative norm) Let $1<p<\infty$, and let $v \in W_{0}^{1, p}(\Omega)$. Then there exists a constant $C$ such that

$$
C\|v\|_{p} \leq\|v\|_{-1, p}+\|\nabla v\|_{-1, p}
$$

Lemma 1.3 (See [9]) Let $v, w \in W^{1, p}(\Omega)$. For each $2<p<\infty$, there exists a constant $C^{\prime}=$ $C^{\prime}(p)>0$ such that

$$
\|\boldsymbol{e}(v)-\boldsymbol{e}(w)\|_{p}^{p} \leq C^{\prime} \int_{\Omega}\left(|\boldsymbol{e}(v)|^{p-2} \boldsymbol{e}(v)-|\boldsymbol{e}(w)|^{p-2} \boldsymbol{e}(w)\right)(\boldsymbol{e}(v)-\boldsymbol{e}(w)) .
$$

By using Hölder's inequality and the imbedding inequality, we could arrive at

$$
\|u\|_{m} \leq C(m, r)\|\nabla u\|_{r}+\tilde{C}(m, r)\|u\|_{2}
$$

with

$$
\begin{cases}m \leq 3 r /(3-r) & \text { for } r \in[1,3) \\ \text { for any } m<\infty & \text { for } r=3 \\ m=\infty & \text { for } r>3\end{cases}
$$


Here, $\tilde{C}(m, r)=0$ if $\left.u\right|_{\partial \Omega}=0$ or $\int_{\Omega} u d x=0$. We will also apply the so-called multiplicative inequalities

$$
\|u\|_{q} \leq C(q)\|\nabla u\|_{2}^{\alpha} \cdot\|u\|_{2}^{1-\alpha}+\hat{C}(q)\|u\|_{2}
$$

with

$$
\alpha=3\left(\frac{1}{2}-\frac{1}{q}\right) \in[0,1], \quad q \in[2,6]
$$

If $\left.u\right|_{\partial \Omega}=0$ or $\int_{\Omega} u d x=0$, then $\hat{C}(q)=0$.

Finally, the paper is organized as follows. In Section 2, we focus on the derivation of the priori estimates for the smooth solutions. On the bases of these estimates, in Section 3, we get the existence result with the help of the Galerkin method. The aim of Section 4 is to give the uniqueness criterion.

\section{The priori estimates}

Let $(u, \omega, b)$ be a smooth solution to system (1.1)-(1.3). The goal of this section is to derive some priori estimates about it. In all the following sections, we always assume that $p \geq \frac{5}{2}$ holds.

Setting $\phi=u$ in (1.5), $\psi=\omega$ in (1.6), $\eta=b$ in (1.7), and observing that $\langle(u \cdot \nabla) u, u\rangle=$ $\langle(u \cdot \nabla) \omega, \omega\rangle=\langle(u \cdot \nabla) b, b\rangle=0$, we obtain

$$
\begin{aligned}
& \frac{1}{2} \frac{d}{d t}\|u\|_{2}^{2}+\|\boldsymbol{e}(u)\|_{p}^{p}=\chi\langle\operatorname{rot} \omega, u\rangle+\langle(b \cdot \nabla) b, u\rangle, \\
& \frac{1}{2} \frac{d}{d t}\|\omega\|_{2}^{2}+\mu\|\nabla \omega\|_{2}^{2}+2 \chi\|\omega\|_{2}^{2}=\chi\langle\operatorname{rot} u, \omega\rangle, \\
& \frac{1}{2} \frac{d}{d t}\|b\|_{2}^{2}+\lambda\|\nabla b\|_{2}^{2}=\langle(b \cdot \nabla) u, b\rangle .
\end{aligned}
$$

Adding the identities above, noting that $\langle(b \cdot \nabla) b, u\rangle+\langle(b \cdot \nabla) u, b\rangle=0$, and Korn's inequality (1.8), we get

$$
\begin{aligned}
\frac{1}{2} & \frac{d}{d t}\left(\|u\|_{2}^{2}+\|\omega\|_{2}^{2}+\|b\|_{2}^{2}\right)+\left(C_{p}\|\nabla u\|_{p}^{p}+\mu\|\nabla \omega\|_{2}^{2}+2 \chi\|\omega\|_{2}^{2}+\lambda\|\nabla b\|_{2}^{2}\right) \\
& =\chi\langle\operatorname{rot} \omega, u\rangle+\chi\langle\operatorname{rot} u, \omega\rangle \\
& \leq C \chi\left(\|\nabla \omega\|_{2} \cdot\|u\|_{2}+\|\nabla u\|_{2} \cdot\|\omega\|_{2}\right) \\
& \leq \varepsilon\left(\|\nabla \omega\|_{2}^{2}+\|\nabla u\|_{p}^{p}\right)+C_{\varepsilon}\left(\|\omega\|_{2}^{2}+\|u\|_{2}^{2}\right) \quad(\text { for } p \geq 2) .
\end{aligned}
$$

After choosing $\varepsilon$ properly small, integrating over $(0, t), t \in(0, T]$, the Gronwall's inequality yields that

$$
\sup _{t \in(0, T)}\left(\|u\|_{2}^{2}+\|\omega\|_{2}^{2}+\|b\|_{2}^{2}\right)+\int_{0}^{T}\left(\|\nabla u\|_{p}^{p}+\|\nabla \omega\|_{2}^{2}+\|\nabla b\|_{2}^{2}\right) \leq C_{1}
$$

where $C_{1}$ is a constant depending on the time $T$ and $\left\|u_{0}\right\|_{2},\left\|w_{0}\right\|_{2},\left\|b_{0}\right\|_{2}$. 
Next, we derive the higher order estimates for $\omega$ and $b$. Setting $\psi=-\Delta \omega$ in (1.6), we find

$$
\begin{aligned}
\frac{1}{2} \frac{d}{d t}\|\nabla \omega\|_{2}^{2}+\mu\|\Delta \omega\|_{2}^{2} & =\langle(u \cdot \nabla) \omega, \Delta \omega\rangle+2 \chi\langle\omega, \Delta \omega\rangle-\chi\langle\operatorname{rot} u, \Delta \omega\rangle \\
& \leq \|(u \cdot \nabla) \omega, \Delta \omega\rangle \mid+\varepsilon\|\Delta \omega\|_{2}^{2}+C_{\varepsilon}\left(\|\omega\|_{2}^{2}+\|\nabla u\|_{2}^{2}\right)
\end{aligned}
$$

for the first term on the right hand side, we compute by the divergence free conditions

$$
\begin{aligned}
|\langle(u \cdot \nabla) \omega, \Delta \omega\rangle| & =\left|\sum_{i j k}\left\langle\partial_{k} u_{i} \partial_{i} \omega_{j}, \partial_{k} \omega_{j}\right\rangle\right| \\
& \leq C \int_{\Omega}\left|\nabla u\left\|\left.\nabla \omega\right|^{2} \leq C\right\| \nabla u\left\|_{p} \cdot\right\| \nabla \omega \|_{\frac{2 p}{p-1}}^{2}\right. \\
& \leq C\|\nabla u\|_{p} \cdot\left(\|\nabla \omega\|_{2}^{\frac{2 p-3}{p}} \cdot\|\Delta \omega\|_{2}^{\frac{3}{p}}+\|\nabla \omega\|_{2}^{2}\right) \\
& \leq \varepsilon\|\Delta \omega\|_{2}^{2}+C_{\varepsilon}\|\nabla u\|_{p}^{\frac{2 p}{2 p-3}} \cdot\|\nabla \omega\|_{2}^{2}+C\|\nabla u\|_{p} \cdot\|\nabla \omega\|_{2}^{2},
\end{aligned}
$$

where Hölder's, Young's inequality and (1.9), (1.10) have been used.

Inserting (2.3) into (2.2), choosing $\varepsilon=\frac{\mu}{4}$ and integrating over $(0, t), t \in(0, T]$, we have

$$
\begin{aligned}
& \frac{1}{2}\|\nabla \omega\|_{2}^{2}+\frac{\mu}{2} \int_{0}^{t}\|\Delta \omega\|_{2}^{2} \\
& \quad \leq C \int_{0}^{t}\left(\|\nabla u\|_{p}^{\frac{2 p}{2 p-3}}+\|\nabla u\|_{p}\right) \cdot\|\nabla \omega\|_{2}^{2}+C \int_{0}^{t}\left(\|\omega\|_{2}^{2}+\|\nabla u\|_{2}^{2}\right)+\frac{1}{2}\left\|\nabla \omega_{0}\right\|_{2}^{2} \\
& \quad \stackrel{(2.1)}{\leq} C \int_{0}^{t}\left(\|\nabla u\|_{p}^{\frac{2 p}{2 p-3}}+\|\nabla u\|_{p}\right) \cdot\|\nabla \omega\|_{2}^{2}+\frac{1}{2}\left\|\nabla \omega_{0}\right\|_{2}^{2}+C_{1},
\end{aligned}
$$

since $p \geq \frac{5}{2}$, we have

$$
\frac{2 p}{2 p-3} \leq p
$$

Gronwall's inequality and estimate (2.1) now provide the bound

$$
\sup _{t \in(0, T)}\|\nabla \omega\|_{2}^{2}+\int_{0}^{T}\|\Delta \omega\|_{2}^{2} \leq C_{2}
$$

where $C_{2}$ is a constant depending on the time $T, C_{1}$ and $\left\|\nabla \omega_{0}\right\|_{2}$.

Next, set $\eta=-\Delta b$ in (1.7) to discover

$$
\begin{aligned}
\frac{1}{2} \frac{d}{d t}\|\nabla b\|_{2}^{2}+\lambda\|\Delta b\|_{2}^{2} & =\langle(u \cdot \nabla) b, \Delta b\rangle-\langle(b \cdot \nabla) u, \Delta b\rangle \\
& \leq|\langle(u \cdot \nabla) b, \Delta b\rangle|+|\langle(b \cdot \nabla) u, \Delta b\rangle| .
\end{aligned}
$$

Reasoning similar to (2.3), we could find

$$
|\langle(u \cdot \nabla) b, \Delta b\rangle| \leq \varepsilon\|\Delta b\|_{2}^{2}+C_{\varepsilon}\|\nabla u\|_{p}^{\frac{2 p}{2 p-3}} \cdot\|\nabla b\|_{2}^{2}+C\|\nabla u\|_{p} \cdot\|\nabla b\|_{2}^{2} .
$$


For the second term on the right hand side of (2.7), we compute

$$
\begin{aligned}
& |\langle(b \cdot \nabla) u, \Delta b\rangle| \\
& \quad \leq \varepsilon\|\Delta b\|_{2}^{2}+C_{\varepsilon}\|(b \cdot \nabla) u\|_{2}^{2} \\
& \quad \leq \varepsilon\|\Delta b\|_{2}^{2}+C_{\varepsilon}\|\nabla u\|_{p}^{2} \cdot\|b\|_{\frac{2 p}{p-2}}^{2} \\
& \quad \leq \varepsilon\|\Delta b\|_{2}^{2}+C_{\varepsilon}\|\nabla u\|_{p}^{2} \cdot\|\nabla b\|_{\frac{6 p}{5 p-6}}^{2} \\
& \quad \leq \varepsilon\|\Delta b\|_{2}^{2}+C_{\varepsilon}\|\nabla u\|_{p}^{2} \cdot\left(\|\nabla b\|_{2}^{\frac{2(2 p-3)}{p}} \cdot\|\Delta b\|_{2}^{\frac{2(3-p)}{p}}+\|\nabla b\|_{2}^{2}\right) \\
& \quad \leq \varepsilon\|\Delta b\|_{2}^{2}+\delta\|\Delta b\|_{2}^{2}+C_{\varepsilon \delta}\|\nabla u\|_{p}^{\frac{2 p}{2 p-3}} \cdot\|\nabla b\|_{2}^{2}+C_{\varepsilon}\|\nabla u\|_{p}^{2} \cdot\|\nabla b\|_{2}^{2}
\end{aligned}
$$

where we have used Hölder's, Young's inequality and (1.9), (1.10). Choosing $\varepsilon$ and $\delta$ properly small, inserting (2.8)-(2.9) into (2.7) and integrating it over $(0, t), t \in(0, T]$, we find

$$
\frac{1}{2}\|\nabla b\|_{2}^{2}+\frac{\lambda}{2} \int_{0}^{t}\|\Delta b\|_{2}^{2} \leq C \int_{0}^{t}\left(\|\nabla u\|_{p}^{\frac{2 p}{2 p-3}}+\|\nabla u\|_{p}^{2}\right) \cdot\|\nabla b\|_{2}^{2}+\frac{1}{2}\left\|\nabla b_{0}\right\|_{2}^{2} .
$$

Observing (2.5) and estimate (2.1), then Gronwall's inequality yields

$$
\sup _{t \in(0, T)}\|\nabla b\|_{2}^{2}+\int_{0}^{T}\|\Delta b\|_{2}^{2} \leq C_{3}
$$

where $C_{3}$ is a constant depending on the time $T, C_{1}$ and $\left\|\nabla b_{0}\right\|_{2}$.

Reasoning analogously to (2.6) and (2.11), it is easy to see that identity (1.6) with $\psi=\omega_{t}$, (1.7) with $\eta=b_{t}$, with the help of (2.6) and (2.11), guarantee the estimate

$$
\int_{0}^{T}\left\|\omega_{t}\right\|_{2}^{2} \leq C_{4}, \quad \int_{0}^{T}\left\|b_{t}\right\|_{2}^{2} \leq C_{5}
$$

where $C_{4}, C_{5}$ are both constants depending only on the time $T$ and some norm of the initial values.

In fact (we here only take $b_{t}$ as an example), set $\eta=b_{t}$ in (1.7), we deduce that

$$
\begin{aligned}
\left\|b_{t}\right\|_{2}^{2}+\frac{\lambda}{2} \frac{d}{d t}\|\nabla b\|_{2}^{2} & \leq\left|\left\langle(b \cdot \nabla) u, b_{t}\right\rangle\right|+\left|\left\langle(u \cdot \nabla) b, b_{t}\right\rangle\right| \\
& \leq \varepsilon\left\|b_{t}\right\|_{2}^{2}+C_{\varepsilon}\left(\|(b \cdot \nabla) u\|_{2}^{2}+\|(u \cdot \nabla) b\|_{2}^{2}\right) .
\end{aligned}
$$

Now, we compute, by using Hölder's, Young's inequality and (1.9), (1.10)

$$
\begin{aligned}
& \|(b \cdot \nabla) u\|_{2}^{2} \leq\|\nabla u\|_{p}^{2} \cdot\|b\|_{\frac{2 p}{p-2}}^{2} \\
& \leq C\left(\|\nabla u\|_{p}^{p}+\|b\|_{\frac{2 p}{p-2}}^{\frac{2 p}{p-2}}\right) \\
& \leq C\left(\|\nabla u\|_{p}^{p}+\|\nabla b\|_{\frac{6 p}{5 p-6}}^{\frac{2 p}{p-2}}\right) \\
& \leq C\|\nabla u\|_{p}^{p}+C\left(\|\nabla b\|_{2}^{\frac{2(2 p-3)}{p-2}} \cdot\|\Delta b\|_{2}^{\frac{2(3-p)}{p-2}}+\|\nabla b\|_{2}^{\frac{2 p}{p-2}}\right)
\end{aligned}
$$


and

$$
\begin{aligned}
\|(u \cdot \nabla) b\|_{2}^{2} & \leq\|u\|_{\frac{3 p}{3-p}}^{2} \cdot\|\nabla b\|_{\frac{6 p}{5 p-6}}^{2} \\
& \leq C\|\nabla u\|_{p}^{2} \cdot\|\nabla b\|_{\frac{6 p}{5 p-6}}^{2} \\
& \leq C\left(\|\nabla u\|_{p}^{p}+\|\nabla b\|_{\frac{6 p}{5 p-6}}^{\frac{2 p}{p-2}}\right) \\
& \leq C\|\nabla u\|_{p}^{p}+C\left(\|\nabla b\|_{2}^{\frac{2(2 p-3)}{p-2}} \cdot\|\Delta b\|_{2}^{\frac{2(3-p)}{p-2}}+\|\nabla b\|_{2}^{\frac{2 p}{p-2}}\right) .
\end{aligned}
$$

Combining (2.13)-(2.15), by choosing $\varepsilon=\frac{1}{2}$, we arrive at

$$
\frac{1}{2}\left\|b_{t}\right\|_{2}^{2}+\frac{\lambda}{2} \frac{d}{d t}\|\nabla b\|_{2}^{2} \leq C\left(\|\nabla u\|_{p}^{p}+\|\nabla b\|_{2}^{\frac{2(2 p-3)}{p-2}} \cdot\|\Delta b\|_{2}^{\frac{2(3-p)}{p-2}}+\|\nabla b\|_{2}^{\frac{2 p}{p-2}}\right),
$$

noting that $p \geq \frac{5}{2}$, so $2(3-p) /(p-2) \leq 2$, and now estimate (2.1), (2.11) and Gronwall's inequality imply the estimate of $b_{t}$ in (2.12).

In the following, we will derive the bound for $u_{t}$. Setting $\eta=u_{t}$ in (1.5), we deduce that

$$
\begin{aligned}
& \left\|u_{t}\right\|_{2}^{2}+\frac{1}{p} \frac{d}{d t}\|\boldsymbol{e}(u)\|_{p}^{p} \\
& \quad \leq\left|\left\langle(b \cdot \nabla) b, u_{t}\right\rangle\right|+\left|\left\langle(u \cdot \nabla) u, u_{t}\right\rangle\right|+\chi\left|\left\langle\operatorname{rot} \omega, u_{t}\right\rangle\right| \\
& \quad \leq \varepsilon\left\|u_{t}\right\|_{2}^{2}+C_{\varepsilon}\left(\|(b \cdot \nabla) b\|_{2}^{2}+\|(u \cdot \nabla) u\|_{2}^{2}+\|\nabla \omega\|_{2}^{2}\right) .
\end{aligned}
$$

Integrating it over $(0, t), t \in(0, T]$, by choosing $\varepsilon=\frac{1}{2}$ and Korn's inequality, we have

$$
\frac{1}{2} \int_{0}^{t}\left\|u_{t}\right\|_{2}^{2}+C_{p}\|\nabla u\|_{p}^{p} \leq C \int_{0}^{t}\left(\|(b \cdot \nabla) b\|_{2}^{2}+\|(u \cdot \nabla) u\|_{2}^{2}\right)+C_{1} .
$$

Now, we compute, by (2.11)

$$
\begin{aligned}
\int_{0}^{t}\|(b \cdot \nabla) b\|_{2}^{2} & \leq \int_{0}^{t}\|b\|_{6}^{2} \cdot\|\nabla b\|_{3}^{2} \\
& \leq C \int_{0}^{t}\|\nabla b\|_{2}^{2} \cdot\left(\|\nabla b\|_{2} \cdot\|\Delta b\|_{2}+\|\nabla b\|_{2}^{2}\right) \\
& \leq C \sup _{\tau \in(0, t)}\|\nabla b\|_{2}^{3} \int_{0}^{t}\|\Delta b\|_{2}+C \sup _{\tau \in(0, t)}\|\nabla b\|_{2}^{4} \cdot t \\
& \leq C_{3}^{4}(1+T), \\
\int_{0}^{t}\|(u \cdot \nabla) u\|_{2}^{2} & \leq \int_{0}^{t}\|u\|_{\frac{2 p}{p-2}}^{2} \cdot\|\nabla u\|_{p}^{2} \leq C \int_{0}^{t}\|\nabla u\|_{p}^{4} .
\end{aligned}
$$

Inserting (2.17)-(2.18) into (2.16), by appealing to Korn's inequality, it follows that

$$
\|\nabla u\|_{p}^{p}+\int_{0}^{t}\left\|u_{t}\right\|_{2}^{2} \leq C \int_{0}^{t}\|\nabla u\|_{p}^{p} \cdot\|\nabla u\|_{p}^{4-p}+C_{6}+C\left\|\nabla u_{0}\right\|_{p}^{p}
$$


where $C_{6}$ depends on $T$ and $C_{3}$. Now, Gronwall's inequality and (2.1) yield that

$$
\sup _{t \in(0, T)}\|\nabla u\|_{p}+\int_{0}^{T}\left\|u_{t}\right\|_{2}^{2} \leq C_{7}
$$

where $C_{7}$ depends on the time $T, C_{1}, C_{3}$ and $\left\|\nabla u_{0}\right\|_{p}$.

\section{Approximate solutions and existence result}

In this section, we show the existence of a weak solution to the system (1.1)-(1.3) via the Galerkin approximations. For this purpose, we take the set $\left\{\phi^{i}\right\}_{i=1}^{\infty}$ formed by the eigenvectors $\phi^{i}, i=1,2, \ldots$, of the Stokes operator and the set $\left\{\psi^{i}\right\}_{i=1}^{\infty}$ formed by the eigenvectors $\psi^{i}, i=1,2, \ldots$, of the Laplace operator. According to the Appendix of [7], the functions $\left\{\phi^{i}\right\}_{i=1}^{\infty}$ form a basis in the space $V_{p}$, and $V_{2} \cap W^{2,2}(\Omega)$. Setting $R_{k}=\operatorname{span}\left\{\phi^{1}, \phi^{2}, \ldots, \phi^{k}\right\}$ and $S_{k}=\operatorname{span}\left\{\psi^{1}, \psi^{2}, \ldots, \psi^{k}\right\}$, we construct the Galerkin approximations $\left\{u^{k}, \omega^{k}, b^{k}\right\}$ being of the form

$$
u^{k}(x, t)=\sum_{i=1}^{k} a_{i}^{k}(t) \phi^{i}(x) ; \quad \omega^{k}(x, t)=\sum_{i=1}^{k} c_{i}^{k}(t) \psi^{i}(x) ; \quad b^{k}(x, t)=\sum_{i=1}^{k} d_{i}^{k}(t) \phi^{i}(x),
$$

where $\boldsymbol{a}^{k}:=\left(a_{1}^{k}, \ldots, a_{k}^{k}\right), \boldsymbol{c}^{k}:=\left(c_{1}^{k}, \ldots, c_{k}^{k}\right), \boldsymbol{d}^{k}:=\left(d_{1}^{k}, \ldots, d_{k}^{k}\right)$ solve the system of ordinary equations

$$
\begin{aligned}
& \left\langle u_{t}^{k}, \phi\right\rangle+\left\langle\left|\boldsymbol{e}\left(u^{k}\right)\right|^{p-2} \boldsymbol{e}\left(u^{k}\right), \boldsymbol{e}(\phi)\right\rangle=\chi\left\langle\operatorname{rot} \omega^{k}, \phi\right\rangle+\left\langle\left(b^{k} \cdot \nabla\right) b^{k}, \phi\right\rangle-\left\langle\left(u^{k} \cdot \nabla\right) u^{k}, \phi\right\rangle \\
& \quad \text { for all } \phi \in R_{k}, \\
& \left\langle\omega_{t}^{k}, \psi\right\rangle+\mu\left\langle\nabla \omega^{k}, \nabla \psi\right\rangle=\chi\left\langle\operatorname{rot} u^{k}, \psi\right\rangle-\left\langle\left(u^{k} \cdot \nabla\right) \omega^{k}, \psi\right\rangle-2 \chi\left\langle\omega^{k}, \psi\right\rangle \\
& \quad \text { for all } \psi \in S_{k}, \\
& \left\langle b_{t}^{k}, \phi\right\rangle+\lambda\left\langle\nabla b^{k}, \nabla \phi\right\rangle=\left\langle\left(b^{k} \cdot \nabla\right) u^{k}, \phi\right\rangle-\left\langle\left(u^{k} \cdot \nabla\right) b^{k}, \phi\right\rangle \\
& \quad \text { for all } \phi \in R_{k} .
\end{aligned}
$$

Moreover, we require that $u^{k}, \omega^{k}, b^{k}$ satisfy the following initial conditions

$$
\left.u^{k}\right|_{t=0}=\sum_{i=1}^{k}\left(u_{0}, \phi^{i}\right) \phi^{i},\left.\quad \omega^{k}\right|_{t=0}=\sum_{i=1}^{k}\left(\omega_{0}, \psi^{i}\right) \psi^{i},\left.\quad b^{k}\right|_{t=0}=\sum_{i=1}^{k}\left(b_{0}, \phi^{i}\right) \phi^{i} .
$$

The local solvability is guaranteed by the Carathéodory theorem, and the global unique solvability follows from the fact that

$$
\begin{aligned}
& \sup _{t \in(0, T)}\left\|u^{k}(t)\right\|_{2}^{2}=\sup _{t \in(0, T)} \sum_{i=1}^{k}\left(a_{i}^{k}(t)\right)^{2} \leq C, \\
& \sup _{t \in(0, T)}\left\|\omega^{k}(t)\right\|_{2}^{2}=\sup _{t \in(0, T)} \sum_{i=1}^{k}\left(c_{i}^{k}(t)\right)^{2} \leq C, \\
& \sup _{t \in(0, T)}\left\|b^{k}(t)\right\|_{2}^{2}=\sup _{t \in(0, T)} \sum_{i=1}^{k}\left(d_{i}^{k}(t)\right)^{2} \leq C
\end{aligned}
$$


with upper bounds $C$ that do not depend on $k$. Moreover, we have for $u^{k}, \omega^{k}$ and $b^{k}$ the same estimates for all norms we have obtained in Section 2. More precisely, we have

$$
\begin{array}{lll}
\sup _{t \in(0, T)}\left\|u^{k}\right\|_{2}, & \sup _{t \in(0, T)}\left\|\nabla u^{k}\right\|_{p}, & \int_{0}^{T}\left\|u_{t}\right\|_{2}^{2} \leq C, \\
\sup _{t \in(0, T)}\left\|\omega^{k}\right\|_{1,2}, & \int_{0}^{t}\left\|\omega^{k}\right\|_{2,2}^{2}, & \int_{0}^{T}\left\|\omega_{t}\right\|_{2}^{2} \leq C, \\
\sup _{t \in(0, T)}\left\|b^{k}\right\|_{1,2}, & \int_{0}^{t}\left\|b^{k}\right\|_{2,2}^{2}, & \int_{0}^{T}\left\|b_{t}\right\|_{2}^{2} \leq C
\end{array}
$$

with a constant $C$ that does not depend on $k$.

Uniform estimates (3.5) imply that there exists a subsequence of $\left\{u^{k}\right\},\left\{\omega^{k}\right\}$ and $\left\{b^{k}\right\}$ (not relabeled) such that

$$
\begin{aligned}
& u^{k} \rightarrow u, \quad \text { weak-* in } L^{\infty}(0, T ; H) \cap L^{\infty}\left(0, T ; V_{p}\right), \\
& \omega^{k} \rightarrow \omega, \quad \text { weakly in } L^{2}\left(0, T ; W^{2,2}(\Omega)\right) \text { and weak-* in } L^{\infty}\left(0, T ; W^{1,2}(\Omega)\right), \\
& b^{k} \rightarrow b, \quad \text { weakly in } L^{2}\left(0, T ; V_{2} \cap W^{2,2}(\Omega)\right) \text { and weak-* in } L^{\infty}\left(0, T ; V_{2}\right), \\
& u_{t}^{k} \rightarrow u_{t}, \quad b_{t}^{k} \rightarrow b_{t}, \quad \text { weakly in } L^{2}(0, T ; H), \\
& \omega_{t}^{k} \rightarrow \omega_{t}, \quad \text { weakly in } L^{2}\left(0, T ; L^{2}(\Omega)\right), \\
& \left|\boldsymbol{e}\left(u^{k}\right)\right|^{p-2} \boldsymbol{e}\left(u^{k}\right) \rightarrow \Lambda, \quad \text { weakly in } L^{p^{\prime}}\left(0, T ; L^{p^{\prime}}(\Omega)\right),
\end{aligned}
$$

where $p^{\prime}=p /(p-1)$. Therefore, by making use of the Aubin-Lions lemma (see Lions [10], Theorem 1.5.1), we have

$$
\begin{aligned}
& u^{k} \rightarrow u, \quad \text { strongly in } L^{2}(0, T ; H), \\
& \omega^{k} \rightarrow \omega, \quad \text { strongly in } L^{2}\left(0, T ; W^{1,2}(\Omega)\right), \\
& b^{k} \rightarrow b, \quad \text { strongly in } L^{2}\left(0, T ; V_{2}\right) .
\end{aligned}
$$

With the convergence above, it is easy to pass to the limit as $k \rightarrow \infty$ in (3.1)-(3.3) to find

$$
\begin{aligned}
& \left\langle u_{t}, \phi\right\rangle+\langle\Lambda, \boldsymbol{e}(\phi)\rangle=\chi\langle\operatorname{rot} \omega, \phi\rangle+\langle(b \cdot \nabla) b, \phi\rangle-\langle(u \cdot \nabla) u, \phi\rangle \\
& \quad \text { for all } \phi \in V_{p}, \\
& \left\langle\omega_{t}, \psi\right\rangle+\mu\langle\nabla \omega, \nabla \psi\rangle=\chi\langle\operatorname{rot} u, \psi\rangle-\langle(u \cdot \nabla) \omega, \psi\rangle-2 \chi\langle\omega, \psi\rangle \\
& \quad \text { for all } \psi \in W^{2,2}(\Omega), \\
& \left\langle b_{t}, \phi\right\rangle+\lambda\langle\nabla b, \nabla \phi\rangle=\langle(b \cdot \nabla) u, \phi\rangle-\langle(u \cdot \nabla) b, \phi\rangle \\
& \quad \text { for all } \phi \in V_{2} .
\end{aligned}
$$

Next, to complete the existence proof, we need to verify that

$$
\Lambda=|\boldsymbol{e}(u)|^{p-2} \boldsymbol{e}(u)
$$


By Lemma 1.3, we have

$$
\begin{aligned}
\left\|\boldsymbol{e}\left(u^{k}\right)-\boldsymbol{e}(u)\right\|_{p}^{p} \leq & C^{\prime} \int_{\Omega}\left(\left|\boldsymbol{e}\left(u^{k}\right)\right|^{p-2} \boldsymbol{e}\left(u^{k}\right)-|\boldsymbol{e}(u)|^{p-2} \boldsymbol{e}(u)\right)\left(\boldsymbol{e}\left(u^{k}\right)-\boldsymbol{e}(u)\right) \\
= & C^{\prime} \int_{\Omega}\left|\boldsymbol{e}\left(u^{k}\right)\right|^{p-2} \boldsymbol{e}\left(u^{k}\right) \boldsymbol{e}\left(u^{k}\right)-\int_{\Omega}|\boldsymbol{e}(u)|^{p-2} \boldsymbol{e}(u)\left(\boldsymbol{e}\left(u^{k}\right)-\boldsymbol{e}(u)\right) \\
& -\int_{\Omega}\left|\boldsymbol{e}\left(u^{k}\right)\right|^{p-2} \boldsymbol{e}\left(u^{k}\right) \boldsymbol{e}(u) \\
\stackrel{(3.1)}{=} \chi\left\langle\operatorname{rot} \omega^{k}, u^{k}\right\rangle+\left\langle\left(b^{k} \cdot \nabla\right) b^{k}, u^{k}\right\rangle-\left\langle u_{t}^{k}, u^{k}\right\rangle & -\int_{\Omega}|\boldsymbol{e}(u)|^{p-2} \boldsymbol{e}(u)\left(\boldsymbol{e}\left(u^{k}\right)-\boldsymbol{e}(u)\right)-\int_{\Omega}\left|\boldsymbol{e}\left(u^{k}\right)\right|^{p-2} \boldsymbol{e}\left(u^{k}\right) \boldsymbol{e}(u) .
\end{aligned}
$$

Considering $\lim _{k \rightarrow \infty}$ of this identity together with (3.6) implies that

$$
\lim _{k \rightarrow \infty}\left\|\boldsymbol{e}\left(u^{k}\right)-\boldsymbol{e}(u)\right\|_{p}^{p} \leq 0
$$

and thus (3.9) follows.

Having the estimates

$$
\|u\|_{L^{\infty}\left(0, T ; W_{0}^{1, p}(\Omega)\right)}, \quad\|\omega\|_{L^{\infty}\left(0, T ; W_{0}^{1,2}(\Omega)\right)}, \quad\|b\|_{L^{\infty}\left(0, T ; W_{0}^{1,2}(\Omega)\right)} \leq C,
$$

we can now introduce the pressure from (1.5). For $t \in(0, T]$, define the functional $F \in$ $W^{-1, p^{\prime}}(\Omega)$ as

$$
\begin{aligned}
\langle F, \xi\rangle:= & \left\langle\operatorname{div}\left(|\boldsymbol{e}(u)|^{p-2} \boldsymbol{e}(u)\right), \xi\right\rangle+\chi\langle\operatorname{rot} \omega, \xi\rangle-\langle b \times \operatorname{rot} b, \xi\rangle \\
& -\langle(u \cdot \nabla) u, \xi\rangle-\left\langle u_{t}, \xi\right\rangle .
\end{aligned}
$$

We have

$$
\langle F, \xi\rangle=0, \quad \forall \xi \in V_{p} \text {, a.e. } t \in(0, T] \text {. }
$$

By using De Rahm's theorem (see [11], Lemma 2.7), we obtain a function $\pi \in L^{p^{\prime}}(\Omega)$ such that

$$
F=-\nabla \pi, \quad \text { a.e. } t \in(0, T] \text {. }
$$

Moreover, due to estimates (3.10),

$$
\|\nabla \pi\|_{W^{-1, p^{\prime}}(\Omega)} \leq C, \quad \text { a.e. } t \in(0, T] .
$$

Then, by Lemma 1.2, there is a generic constant $C$, depending only on the data such that

$$
\|\pi\|_{p^{\prime}} \leq C, \quad \text { a.e. } t \in(0, T]
$$

Now, we complete the proof of the existence part of Theorem 1.1. 


\section{Uniqueness criterion}

Let $\left(u_{1}, \omega_{1}, b_{1}\right)$ and $\left(u_{2}, \omega_{2}, b_{2}\right)$ be both solutions of the problem. Then, for their difference $\bar{u}=u_{1}-u_{2}, \bar{\omega}=\omega_{1}-\omega_{2}, \bar{b}=b_{1}-b_{2}$, we have

$$
\begin{aligned}
& \left\langle\bar{u}_{t}, \phi\right\rangle+\left\langle\left|\boldsymbol{e}\left(u_{1}\right)\right|^{p-2} \boldsymbol{e}\left(u_{1}\right)-\left|\boldsymbol{e}\left(u_{2}\right)\right|^{p-2} \boldsymbol{e}\left(u_{2}\right), \boldsymbol{e}(\phi)\right\rangle \\
& =\chi\langle\operatorname{rot} \bar{\omega}, \phi\rangle+\left\langle\left(b_{1} \cdot \nabla\right) \bar{b}, \phi\right\rangle \\
& \quad+\left\langle(\bar{b} \cdot \nabla) b_{2}, \phi\right\rangle-\left\langle\left(u_{1} \cdot \nabla\right) \bar{u}, \phi\right\rangle-\left\langle(\bar{u} \cdot \nabla) u_{2}, \phi\right\rangle \quad \text { for all } \phi \in V_{p}, \\
& \left\langle\bar{\omega}_{t}, \psi\right\rangle+\mu\langle\nabla \bar{\omega}, \nabla \psi\rangle+2 \chi\langle\bar{\omega}, \psi\rangle=\chi\langle\operatorname{rot} \bar{u}, \psi\rangle-\left\langle\left(u_{1} \cdot \nabla\right) \bar{\omega}, \psi\right\rangle-\left\langle(\bar{u} \cdot \nabla) \omega_{2}, \psi\right\rangle \\
& \quad \text { for all } \psi \in W_{0}^{1,2}(\Omega), \\
& \left\langle\bar{b}_{t}, \eta\right\rangle+\lambda\langle\nabla \bar{b}, \nabla \eta\rangle=\left\langle\left(b_{1} \cdot \nabla\right) \bar{u}, \eta\right\rangle+\left\langle(\bar{b} \cdot \nabla) u_{2}, \eta\right\rangle-\left\langle\left(u_{1} \cdot \nabla\right) \bar{b}, \eta\right\rangle-\left\langle(\bar{u} \cdot \nabla) b_{2}, \eta\right\rangle \\
& \quad \text { for all } \eta \in V_{2} .
\end{aligned}
$$

Taking $\phi=\bar{u}$ in (4.1), by Lemma 1.3 and the fact that $\left\langle\left(u_{1} \cdot \nabla\right) \bar{u}, \bar{u}\right\rangle=0$ and $p>2$, we obtain

$$
\begin{aligned}
\frac{1}{2} \frac{d}{d t}\|\bar{u}\|_{2}^{2}+C_{p}\|\nabla \bar{u}\|_{p}^{p} & \leq \chi\langle\operatorname{rot} \bar{\omega}, \bar{u}\rangle+\left\langle(\bar{b} \cdot \nabla) b_{2}, \bar{u}\right\rangle-\left\langle(\bar{u} \cdot \nabla) u_{2}, \bar{u}\right\rangle+\left\langle\left(b_{1} \cdot \nabla\right) \bar{b}, \bar{u}\right\rangle \\
& \equiv \sum_{i=1}^{3} J_{i}+\left\langle\left(b_{1} \cdot \nabla\right) \bar{b}, \bar{u}\right\rangle
\end{aligned}
$$

for each $J_{i}, i=1,2,3$, it follows from Hölder's, Young's inequality and (1.9), (1.10) that

$$
\begin{aligned}
J_{1} & \leq \chi|\langle\operatorname{rot} \bar{\omega}, \bar{u}\rangle| \leq \chi\|\nabla \bar{\omega}\|_{2} \cdot\|\bar{u}\|_{2} \leq \varepsilon\|\nabla \bar{\omega}\|_{2}^{2}+C_{\varepsilon}\|\bar{u}\|_{2}^{2}, \\
J_{2} & \leq \mid\left\langle(\bar{b} \cdot \nabla) b_{2}, \bar{u}\|\mid \leq\| \nabla b_{2}\left\|_{6} \cdot\right\| \bar{b}\left\|_{3} \cdot\right\| \bar{u}\left\|_{2} \leq C\left(\left\|\Delta b_{2}\right\|_{2}+\left\|\nabla b_{2}\right\|_{2}\right) \cdot\right\| \nabla \bar{b}\left\|_{2} \cdot\right\| \bar{u} \|_{2}\right. \\
& \leq \varepsilon\|\nabla \bar{b}\|_{2}^{2}+C_{\varepsilon}\left(\left\|\Delta b_{2}\right\|_{2}^{2}+\left\|\nabla b_{2}\right\|_{2}^{2}\right) \cdot\|\bar{u}\|_{2}^{2}, \\
J_{3} & \leq \mid\left\langle(\bar{u} \cdot \nabla) u_{2}, \bar{u}\|\leq\| \nabla u_{2}\left\|_{p} \cdot\right\| \bar{u}\left\|_{\frac{2 p}{p-1}}^{2} \leq\right\| \nabla u_{2}\left\|_{p} \cdot\right\| \bar{u}\left\|_{2}^{\frac{2 p-3}{p}} \cdot\right\| \nabla \bar{u} \|_{2}^{\frac{3}{p}}\right. \\
& \leq \varepsilon\|\nabla \bar{u}\|_{2}^{2}+C_{\varepsilon}\left\|\nabla u_{2}\right\|_{p}^{\frac{2 p}{2 p-3}} \cdot\|\bar{u}\|_{2}^{2},
\end{aligned}
$$

so, we have

$$
\begin{aligned}
& \frac{1}{2} \frac{d}{d t}\|\bar{u}\|_{2}^{2}+C_{p}\|\nabla \bar{u}\|_{p}^{p} \\
& \quad \leq \varepsilon\left(\|\nabla \bar{u}\|_{2}^{2}+\|\nabla \bar{\omega}\|_{2}^{2}+\|\nabla \bar{b}\|_{2}^{2}\right) \\
& \quad+C_{\varepsilon}\left(1+\left\|\nabla b_{2}\right\|_{2}^{2}+\left\|\Delta b_{2}\right\|_{2}^{2}+\left\|\nabla u_{2}\right\|_{p}^{\frac{2 p}{2 p-3}}\right) \cdot\|\bar{u}\|_{2}^{2}+\left\langle\left(b_{1} \cdot \nabla\right) \bar{b}, \bar{u}\right\rangle .
\end{aligned}
$$

Taking $\psi=\bar{\omega}$ in (4.2) and noting that $\left\langle\left(u_{1} \cdot \nabla\right) \bar{\omega}, \bar{\omega}\right\rangle=0$, it follows

$$
\begin{aligned}
\frac{1}{2} \frac{d}{d t}\|\bar{\omega}\|_{2}^{2}+\mu\|\nabla \bar{\omega}\|_{2}^{2}+2 \chi\|\bar{\omega}\|_{2}^{2} & =\chi\langle\operatorname{rot} \bar{u}, \bar{\omega}\rangle-\left\langle(\bar{u} \cdot \nabla) \omega_{2}, \bar{\omega}\right\rangle \\
& \equiv \sum_{i=1}^{2} I_{i}
\end{aligned}
$$


and for $I_{i}, i=1,2$,

$$
\begin{aligned}
I_{1} & \leq \chi\|\nabla \bar{u}\|_{2} \cdot\|\bar{\omega}\|_{2} \leq \varepsilon\|\nabla \bar{u}\|_{2}^{2}+C_{\varepsilon}\|\bar{\omega}\|_{2}^{2}, \\
I_{2} & \leq\left\|\nabla \omega_{2}\right\|_{6} \cdot\|\bar{\omega}\|_{3} \cdot\|\bar{u}\|_{2} \leq C\left(\left\|\Delta \omega_{2}\right\|_{2}+\left\|\nabla \omega_{2}\right\|_{2}\right) \cdot\|\nabla \bar{\omega}\|_{2} \cdot\|\bar{u}\|_{2} \\
& \leq \varepsilon\|\nabla \bar{\omega}\|_{2}^{2}+C_{\varepsilon}\left(\left\|\Delta \omega_{2}\right\|_{2}^{2}+\left\|\nabla \omega_{2}\right\|_{2}^{2}\right) \cdot\|\bar{u}\|_{2}^{2},
\end{aligned}
$$

thus, we obtain

$$
\begin{aligned}
\frac{1}{2} \frac{d}{d t}\|\bar{\omega}\|_{2}^{2}+\mu\|\nabla \bar{\omega}\|_{2}^{2} \leq & \varepsilon\left(\|\nabla \bar{u}\|_{2}^{2}+\|\nabla \bar{\omega}\|_{2}^{2}\right)+C_{\varepsilon}\|\bar{\omega}\|_{2}^{2} \\
& +C_{\varepsilon}\left(\left\|\Delta \omega_{2}\right\|_{2}^{2}+\left\|\nabla \omega_{2}\right\|_{2}^{2}\right) \cdot\|\bar{u}\|_{2}^{2} .
\end{aligned}
$$

Similarly, by taking $\eta=\bar{b}$ in (4.3), reasoning analogous as above, we could get

$$
\begin{aligned}
\frac{1}{2} \frac{d}{d t}\|\bar{b}\|_{2}^{2}+\lambda\|\nabla \bar{b}\|_{2}^{2} \leq & \varepsilon\|\nabla \bar{b}\|_{2}^{2}+C_{\varepsilon}\left(\left\|\Delta b_{2}\right\|_{2}^{2}+\left\|\nabla b_{2}\right\|_{2}^{2}\right) \cdot\|\bar{u}\|_{2}^{2} \\
& +C_{\varepsilon}\left\|\nabla u_{2}\right\|_{p}^{\frac{2 p}{2 p-3}} \cdot\|\bar{b}\|_{2}^{2}+\left\langle\left(b_{1} \cdot \nabla\right) \bar{u}, \bar{b}\right\rangle .
\end{aligned}
$$

Adding (4.4)-(4.6) and observing that $\left\langle\left(b_{1} \cdot \nabla\right) \bar{b}, \bar{u}\right\rangle+\left\langle\left(b_{1} \cdot \nabla\right) \bar{u}, \bar{b}\right\rangle=0$, after choosing $\varepsilon$ properly small, we finally get

$$
\begin{aligned}
& \frac{1}{2} \frac{d}{d t}\left(\|\bar{u}\|_{2}^{2}+\|\bar{\omega}\|_{2}^{2}+\|\bar{b}\|_{2}^{2}\right)+C\left(\|\nabla \bar{u}\|_{2}^{2}+\|\nabla \bar{\omega}\|_{2}^{2}+\|\nabla \bar{b}\|_{2}^{2}\right) \\
& \quad \leq C F(t) \cdot\left(\|\bar{u}\|_{2}^{2}+\|\bar{\omega}\|_{2}^{2}+\|\bar{b}\|_{2}^{2}\right)
\end{aligned}
$$

with

$$
F(t)=1+\left\|\nabla b_{2}\right\|_{2}^{2}+\left\|\Delta b_{2}\right\|_{2}^{2}+\left\|\nabla u_{2}\right\|_{p}^{\frac{2 p}{2 p-3}}+\left\|\nabla \omega_{2}\right\|_{2}^{2}+\left\|\Delta \omega_{2}\right\|_{2}^{2} .
$$

Since $2 p /(2 p-3) \leq p$ for $p \geq \frac{5}{2}$, then Gronwall's inequality and the estimates obtained in Section 2 yield that

$$
\bar{u}=\bar{\omega}=\bar{b}=0 \quad \text { for } t \in[0, T] .
$$

This completes the proof of the theorem.

\section{Competing interests}

The author declares that they have no competing interests.

\section{Authors' contributions}

The author completed the paper. The author read and approved the final manuscript.

\section{Acknowledgements}

The author would like to thank the referees for valuable comments and suggestions for improving this paper. This work was partially supported by the National NSF (Grant No. 10971080) of China. 
References

1. Eringen, AC: Theory of micropolar fluids. J. Math. Mech. 16, 1-18 (1966)

2. Galdi, GP, Rionero, S: A note on the existence and uniqueness of solutions of the micropolar fluid equations. Int J. Eng. Sci. 15, 105-108 (1977)

3. Rojas-Medar, MA: Magneto-micropolar fluid motion: existence and uniqueness of strong solution. Math. Nachr. 188, 301-319 (1997)

4. Ortega-Torres, EE, Rojas-Medar, MA: Magneto-micropolar fluid motion: global existence of strong solutions. Abstr. Appl. Anal. 4, 109-125 (1999)

5. Rojas-Medar, MA, Boldrini, JL: Magneto-micropolar fluid motion: existence of weak solutions. Rev. Mat. Complut. 11, 443-460 (1998)

6. Gunzburger, MD, Ladyzhenskaya, OA, Peterson, JS: On the global unique solvability of initial-boundary value problems for the coupled modified Navier-Stokes and Maxwell equations. J. Math. Fluid Mech. 6, 462-482 (2004)

7. Málek, J, Něcas, J, Rokyta, M, Ružička, M: Weak and Measure-Valued Solutions to Evolutionary PDEs. Chapman \& Hall, London (1996)

8. Bellout, H, Bloom, F, Nečas, J: Solutions for incompressible non-Newtonian fluids. C. R. Math. Acad. Sci. Paris, Sér. I 317, 795-800 (1993)

9. Ebmeyer, C, Urbano, JM: Quasi-steady Stokes flow of multiphase fluids with shear-dependent viscosity. Eur. J. Appl. Math. 18, 417-434 (2007)

10. Lions, JL: Quelques méthodes de Résolution de Problèmes aux limites non Linéaires. Dunod, Paris (1969)

11. Amrouche, C, Girault, V: Decomposition of vector spaces and application to the Stokes problem in arbitrary dimension. Czechoslov. Math. J. 44, 109-140 (1994)

doi:10.1186/1687-2770-2013-182

Cite this article as: Wang: Unique solvability for the non-Newtonian magneto-micropolar fluid. Boundary Value Problems 2013 2013:182

\section{Submit your manuscript to a SpringerOpen ${ }^{\ominus}$ journal and benefit from:}

- Convenient online submission

- Rigorous peer review

- Immediate publication on acceptance

- Open access: articles freely available online

- High visibility within the field

- Retaining the copyright to your article 